University of Nebraska - Lincoln

DigitalCommons@University of Nebraska - Lincoln

20th \& 21st Century French and Francophone Modern Languages and Literatures, Department Studies International Colloquium

\title{
L’art féminin, un cri d’espoir pour l'humanité
}

\author{
Zohra Lhioui
}

Follow this and additional works at: https://digitalcommons.unl.edu/ffsc2020

Part of the Comparative Literature Commons, French and Francophone Literature Commons, and the Other French and Francophone Language and Literature Commons

This Presentation is brought to you for free and open access by the Modern Languages and Literatures, Department of at DigitalCommons@University of Nebraska - Lincoln. It has been accepted for inclusion in 20th \& 21st Century French and Francophone Studies International Colloquium by an authorized administrator of DigitalCommons@University of Nebraska - Lincoln. 


\title{
L'art féminin, un cri d'espoir pour l'humanité
}

\author{
Professeure Zohra Lhioui \\ Université Moulay Ismaïl \\ Meknès. Maroc \\ Z.1hioui@umi.ac.ma
}

\section{Résumé}

A travers une étude réalisée au Maroc, dans la Région de Meknès-Tafilalet (Centre-Est du royaume), et qui a été couronnée par la publication d'un ouvrage d'art portant le titre Femmes artistes de Meknès-Tafilalet (voir couverture jointe à cette proposition de communication), il ressort que l'art chez les femmes de cette région n'est pas une simple question de créativité et de passion, il est aussi un moyen esthétique d'exprimer la richesse du patrimoine, la culture de leur société et leur attachement à la nature, à l'environnement, bref à la vie tout court.

Que ce soit par la danse, le stylisme, la broderie, la peinture, la photo, la poésie ou l'artisanat, cent femmes expriment dans ce bel ouvrage leur soif de liberté, d'émancipation et de réappropriation de leur corps, elles révèlent également leur féminité et célèbrent la beauté de la nature et l'amour de la vie.

La question qui se pose est la suivante : comment l'art, sous toutes ses formes, devient-il chez les femmes un moyen d'épanouissement et un cri d'espoir pour l'humanité et l'environnement?

Cette communication, en plus de présenter un compte rendu de l'ouvrage d'art Femmes artistes de Meknès-Tafilalet dont j'ai coordonné la réalisation et la publication, tentera d'apporter quelques éclairages sur le rapport entre l'art au féminin et le combat des femmes pour un monde meilleur et une société juste et respectueuse de la nature.

Mots clés : Femmes, Art, Liberté, Espoir, Nature, Environnement, Maroc

Notice biographique : Zohra Lhioui est professeure de littérature comparée. Elle a publié plusieurs articles sur J.L.Borges et sur la question du genre dans des revues nationales et internationales, elle a également coordonné des publications sur le genre. Elle est actuellement coordinatrice de l'Equipe Pluridisciplinaire de Recherche sur la Femme (TANIT) de l'Université Moulay Ismaïl, elle est aussi directrice du Centre de Recherche Scientifique en Littérature et Sciences Humaines de la faculté des Lettres de l'université Moulay Ismaïl. Elle a coordonnée plusieurs publications sur le genre dont essentiellement :

Art au féminin et Code de la nationalité : un nouveau pas vers l'égalité, Série Actes de colloques, $\mathrm{n}^{\circ}$ 28, 2011.

Femme, Education et Développement, Série «Etudes et Recherches n³0/2011», co-publication Faculté des Lettres de Meknès et Faculté de l’Éducation de Séville (Espagne), 2011

- Femmes artistes de Meknès-Tafilalet, Ed. Maison de la Presse, Meknès, 2013.

- Publication des actes de la journée d'étude sur Fatima Mernissi« Rêves d'une femme ", Publication de la Faculté des Lettres et des Sciences humaines, 2016. 
«La violence fondée sur le genre au Maroc et en Espagne : milieu scolaire et universitaire ", Publication de la Faculté des Lettres et des Sciences humaines, 2018.

- $\quad$ Publication des actes du colloque international sur «Manuels scolaires et culture de l'égalité des genres ", Publication de l'Université Moulay Ismaïl, 2018.

\section{Introduction}

Femmes Artistes de Meknès Tafilalet est le fruit d'un travail collectif de TANIT ${ }^{1}$, Equipe Pluridisciplinaire de Recherche sur la Femme, relevant de la Faculté des Lettres et des Sciences Humaines, financé par l'Université Moulay Ismail dans le cadre d'un programme d'appui à la recherche.

Paru en décembre 2013, cet ouvrage a eu le privilège d'être remarquablement préfacé par une éminente artiste peintre et sculptrice marocaine Ahlam Lemseffer, et coordonnée par l'enseignante chercheure et coordinatrice de l'équipe de recherche Tanit. Zohra Lhioui.

Il s'agit certes d'un beau livre en papier glacé mais il est plus que cela. Ses auteures ont tenu à valoriser esthétiquement les créations de quelques femmes artistes de l'ex-région MeknèsTafilalet. L'objectif majeur de cette étude est de les sortir d'un relatif anonymat, de l'ombrepour certaines- et de les hisser à la notoriété par l'Université.

L'émotion nous saisit en feuilletant l'ouvrage de TANIT qui révèle les efforts de ces femmes pour s'exprimer; elles veulent dire au moyen de leur création qu'elles existent, qu'elles aiment ce monde dans lequel nous vivons et qu'elles y apportent, chacune à sa façon, une touche personnelle pour l'embellir et le préserver.

L'ouvrage présente d'abord les résultats de l'enquête sur l'activité artistique de plus d'une centaine de femmes résidant dans l'ex-Région de Meknès Tafilalet, située au Nord du Maroc et qui s'étend à l'Est et au Sud. Après un survol de leurs profils social et artistique, l'équipe TANIT s'est intéressée aux aspects relatifs à leur activité artistique. La diversité de l'échantillon est surprenante par sa richesse. Ainsi l'étude a porté sur leur milieu de résidence, la langue parlée arabe et/ou amazigh, leur situation familiale, leur domaine artistique, et la

\footnotetext{
${ }^{1}$ C'est le nom de la Déesse Carthaginoise de la fertilité, "La Grande Dame de Carthage", compagne inséparable du Dieu Ba'alHammôn. Elle était considérée, au même titre que Ba'al, comme la protectrice de la Cité. La séduction qu'a exercée toute la symbolique dégagée par ce personnage féminin mythique a motivé notre choix de baptiser cette Equipe de Recherche Pluridisciplinaire sur la Femme TANIT. D'une part, parce que la Déesse TANIT régnait côte à côte avec l'homme, et d'autre part, parce qu'elle était sa compagne inséparable.
} 
catégorie professionnelle. L'ouvrage renferme, illustrations à l'appui, des créations artistiques de trente-quatre femmes de cette ex-région, avec leurs photographies en médaillons. Création artistique qui embrasse la peinture, le tissage, la sculpture, le stylisme, la broderie, le dessin, les arts décoratifs, le chant, la poésie, la musique et bien d'autres domaines où brille la femme de Meknès, du Moyen Atlas et du Tafilalet.

Ce travail est envisagé en quatre sections qui correspondent chacune à l'un des pouvoirs multiples dont l'art investi par les femmes dans un contexte socio-culturel marocain au XXIe siècle. Le corpus est bien évidemment l'ouvrage sur lequel porte cette étude et que je citerai assez souvent pour conforter mes réflexions et analyses.

D'aucuns y verront des ressemblances indéniables avec la situation des femmes artistes dans les autres cultures et sociétés, ce qui est d'ailleurs vrai, cependant, dans certaines sociétés musulmanes où le fondamentalisme joue des coudes pour restreindre de plus en plus la liberté et l'épanouissement des femmes, l'art des femmes devient un élan de militantisme, une lutte pour l'existence.

Les extrémistes religieux ont de tout temps pris pour cible l'art en général et l'art au féminin en particulier, conscients des pouvoirs immenses de l'art qui représentent un danger pour les intérêts et l'idéologie du fanatisme religieux.

Lors de la réalisation de cet ouvrage, et plus précisément à l'occasion des entretiens avec les femmes artistes dont certains extraits seront cités ici, j'ai été fascinée par leur attachement à leurs productions, partie intégrante de leur identité, par leurs attentes et l'impact espéré. Quels sont donc les pouvoirs de l'art qui se dégagent des créations et des révélations de ces artistes? Quelques recoupements s'avèrent nécessaires entre une lecture de leurs productions et le contenu de leurs propos.

\section{Les pouvoirs de l'art des femmes}

\section{1- Se faire entendre, exister}

Ces multiples formes d'art que présente cet ouvrage, mêlant le rêve à la réalité, renferment, sans exception, le désir des femmes artistes de se faire entendre, de prendre la parole, faisant de l'art leur voix, leur droit à l'existence, à la vie tout court. C'est ce que révèle l'enquête réalisée.

Bien que pour une grande majorité de femmes $(63,4 \%)$ l'activité reste une source de revenu, elle n'en est pas moins pour un grand nombre d'entre elles un hobby $(53,5 \%)$ et une passion pour d'autres $(41,6 \%)$. Mais, dans aucun cas cette activité n'est considérée comme un passetemps $(0 \%)$. Ainsi ces résultats font ressortir l'importance de l'activité artistique pour ces 
femmes, qui est une activité valorisante à tous points de vue : économique, social, personnel et parfois même psychologique. ${ }^{2}$

Comme l'exprime avec brio la préfacière du livre, la peintre et sculptrice Ahlam Lemseffer : «Le thème de la femme qui revient chez nombre de ces artistes est révélateur de leur condition et de leur soif d'exister et de rompre avec la solitude, l'incompréhension et l'angoisse. $\|^{3}$

Exister, mais aussi puiser sa force, son énergie dans l'art ; l'acte de créer chez ces femmes devient une activité presque vitale, c'est leur espace intime, un refuge quand le quotidien devient intolérable; «briser la monotonie du quotidien», «oublier le poids des devoirs familiaux et professionnels », ce sont des propos qui reviennent souvent chez l'artiste peintre Leïla Benyahia ${ }^{4}$.

Ces femmes artistes dont certaines sont analphabètes aspirent tout simplement à la lumière, à la visibilité. Fatima Benradouane est l'exemple parfait de la femme marocaine, analphabète, d'un milieu modeste, femme au foyer, mère de six enfants (deux filles et quarte garçons) ignorant tout de l'histoire de l'art, des techniques esthétiques. Elle jette son dévolu sur la peinture à un âge mûr, d'une manière presque instinctive. Quand on lui pose la question : pourquoi la peinture? Elle répond ne pas avoir d'explication. Elle manie le pinceau par amour, habitée par la rage de peindre au point d'économiser sur l'argent des provisions pour se procurer le matériel nécessaire. La peinture est son monde dans le sens propre du terme puisqu'elle a aménagé l'unique salon de sa petite maison en salle d'exposition. (Voir photo en annexes)

L'univers magique de «Khalti Fatima» est un foisonnement fabuleux de personnages, de formes et d'objets aux couleurs vives sans aucun respect des perspectives ni des couleurs, un monde né de l'intuition, du rêve d'une femme autodidacte, prête à tout sacrifier pour sa passion. Son style de peinture nous fait inéluctablement penser à feu Chaâbia qu'elle avoue connaître vaguement.

Grâce au soutien de son mari et de tout son entourage, Fatima réalise son rêve le plus cher, celui de sortir de l'ombre et de devenir une artiste peintre à part entière. ${ }^{5}$

Grâce à ses tableaux de style naïf, elle est devenue la fierté de sa ville Midelt dont les habitants lui témoignent respect et reconnaissance.

\footnotetext{
${ }^{2}$ Les Femmes artistes de Meknès-Tafilalet, Publications de l'Université Moulay Ismaïl, Ed. Maison de la Presse, Meknès, 2013, p.20.

${ }^{3}$ Ibid., voir la préface p.13.

${ }^{4}$ Ibid., p. 40.

${ }^{5}$ lbid., p.39.
} 


\section{2- Revendiquer sa liberté, s'approprier son corps}

Le corps mutilé, torturé, déformé, souffrant chez l'artiste Naïma Zryouil résume parfaitement le malaise des femmes dans la société, l'incommunicabilité entre les hommes et les femmes :

Privées du regard et des mains, ces formes humaines paraissent avoir des difficultés à communiquer, comme le montre ce tableau où deux corps se trouvent face à face. Ce monde énigmatique baigne dans le silence et l'angoisse et suggère la dimension tragique de l'homme, régie par le sentiment d'étrangeté, de solitude intérieure et d'incommunicabilité. Tout ceci se trouve accentué par le choix de couleurs où prédominent le noir et le gris foncé aux dépens des couleurs chaudes et lumineuses. ${ }^{6}$

En plus d'exprimer le mal être des femmes, l'art devient aussi leur espace de liberté, d'émancipation, de revendications non seulement de leur droit à l'existence mais d'un monde meilleur, un monde où règnent l'harmonie et la beauté. Dans une société arabo-musulmane où on a tendance à nier le corps, à le voiler, à le réprimer, à lui interdire même d'investir librement l'espace public, l'art devient le lieu de l'expression du corps dans tous ses états, le corps libéré de ses chaînes, de ses voiles et masques. Zahra Awwaje, artiste peintre de la ville de Meknès, trouve l'audace de

\footnotetext{
«montrer les corps dans leur nudité, une nudité pudique qui, sans en dévoiler l'intimité, les couvre de mystère et les révèle dans leur beauté et leur grâce. Cette technique du flou confère à son «nu » une touche propre à elle. Dans son choix de peindre le nu, Zahra réussit à briser les tabous et à célébrer le corps féminin, invitant les gens à changer leur vision et leur rapport à ce corps, souvent réduit à un objet de désir, de consommation.7
}

Le tableau de la couverture peut être interprété dans le même sens même si le corps n'y est représenté que par une partie. Seulement, comment ne pas y voir une figure de style, la synecdoque en l'occurrence, qui consiste à utiliser la partie pour le tout.

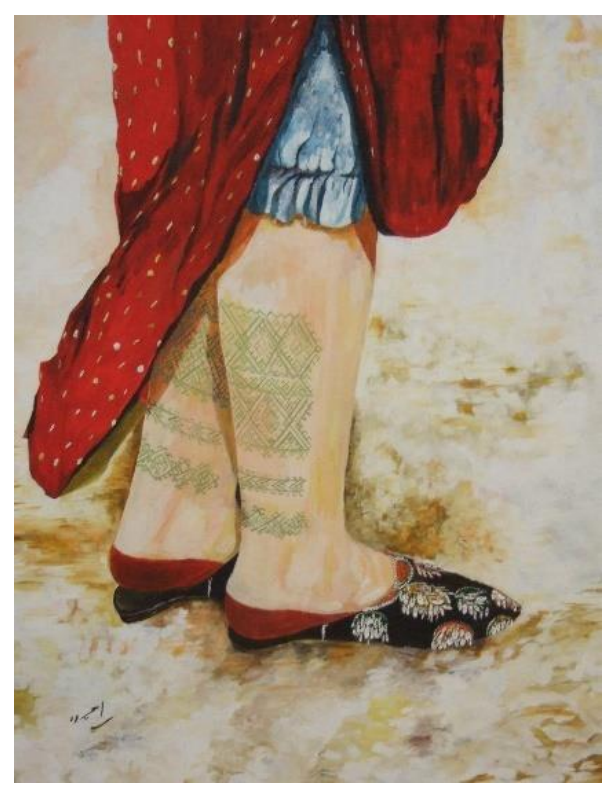

\footnotetext{
${ }^{6}$ Les femmes artistes de Meknès-Tafilalet, op. cit., p.72.

7 Ibid., p.32.
} 
Ce tableau a été choisi pour sa charge symbolique féminine et culturelle mais également pour sa beauté et le choix esthétique des formes et couleurs. Il s'agit de la représentation d'une partie du corps de la femme, en l'occurrence les mollets et les pieds, partie dont l'érotisme n'est un mystère pour personne ; des mollets à la peau blanche, d'une rondeur splendide, soigneusement tatoués, laissant voir des figures géométriques d'une perfection remarquable. Les pieds sont subtilement peints au henné rouge foncé et enfilés dans de jolies babouches brodées de fleurs. On entrevoit également un sarouel bleu ciel s'arrêtant juste au-dessous du genou droit, un pan de caftan rouge grenat éclaboussé de petites taches blanches et descendant jusqu'au pied gauche alors que le pan de devant est rabattu dévoilant le bout du saroual et les mollets tatoués.

Cette peinture qui associe harmonieusement féminité, érotisme et patrimoine culturel exprime un regard intelligent et libre d'une artiste peintre, Rahma El Moumen, qui, sans montrer le corps dans sa nudité, le suggère avec finesse et dextérité.

La nudité partielle (Rahma El Moumen) ou pudique (Zahra Awwaj) ne présente nullement les femmes en tant qu'objets sexuels prêts à la consommation. La nudité devient chez ces artistes une forme d'appropriation de leur corps, de quête de liberté, de reconstruction de leur image.

\section{3- Euvrer pour un monde meilleur, célébrer la nature, la beauté et la joie de vivre}

Nous remarquons que pour certaines artistes, la célébration de la nature est au centre de leurs créations et donc de leurs préoccupations. Ainsi, Goulnara Ichmetova-Sebati, artiste peintre d'origine russe, révèle que

la peinture des paysage, la représentation de la nature restent son domaine de prédilection. En effet, Goulnara semble préférer la nature et représenter les sentiments éprouvés selon les saisons : le printemps ou la renaissance de la nature ; la fonte des neiges en Russie annonçant l'arrivée du printemps, l'éclosion des roses, un paysage de Chefchaouen ${ }^{8}$, Sahrij Souani', etc.

Souvent dans le choix des couleurs se lit le souci de faire de la peinture un écho de la nature, du cosmos, c'est ce qui est explicitement exprimé dans les toiles de la peintre et poétesse Chama Ami (voir tableaux 1 et 2 en annexe)

Il est vrai que peinture, collage et patchwork sont trois techniques différentes mais dans le travail de cette artiste peintre, ils sont unis par la récurrence des formes géométriques et la prédominance de certaines couleurs, le bleu, le vert, le jaune, le rouge, le mauve et le marron, couleur de la terre. Ces couleurs qui symbolisent les éléments fondamentaux du Cosmos (l'eau, le feu, la terre...), rappellent-elles le lien éternel entre l'homme et l'univers ? $^{10}$

\footnotetext{
${ }^{8}$ Ville du nord du Maroc, très prisée par les touristes.

${ }^{9}$ Bassin à Meknès, construit au XVIle siècle par le Sultan Moulay Ismaïl

${ }^{10}$ Femmes artistes de Meknès-Tafilalet, op.cit., p.29.
} 
Et voici comment cette artiste définit elle-même sa peinture :

Une toile est avant tout un objet à voir, un objet-message, et si message il y a, c'est de développer le sens du beau chez l'autre et l'initier à lire, à déchiffrer ce monde des couleurs qui, loin d'être une activité ludique, est un moyen d'expression à travers et par lequel l'artiste essaie d'exprimer sa vision du monde ainsi que son rapport à l'autre et aux choses.

Représenter le monde et sa magie sous une forme féérique chez la majorité des artistes de cet ouvrage traduit l'aspiration des femmes à un monde harmonieux où règnent la beauté, la sérénité et le bien-être. C'est ce qui est représenté par une végétation généreuse, des fleurs aux multiples couleurs, une nature luxuriante, un environnement qui respire la santé. L'instinct de protection de la nature est perceptible chez les femmes plus que chez les hommes grâce à leur pouvoir nourricier. Détruire la nature les impacte directement dans leurs fonctions traditionnelles : nourrir, soigner et protéger. Porteuses de vie, l'art devient ainsi pour elles une forme d'engagement dans le combat pour l'écologie, pour la préservation de l'environnement, de la vie. Peindre la nature, même si cela semble quelque peu idyllique, devient l'expression de leur amour de la nature.

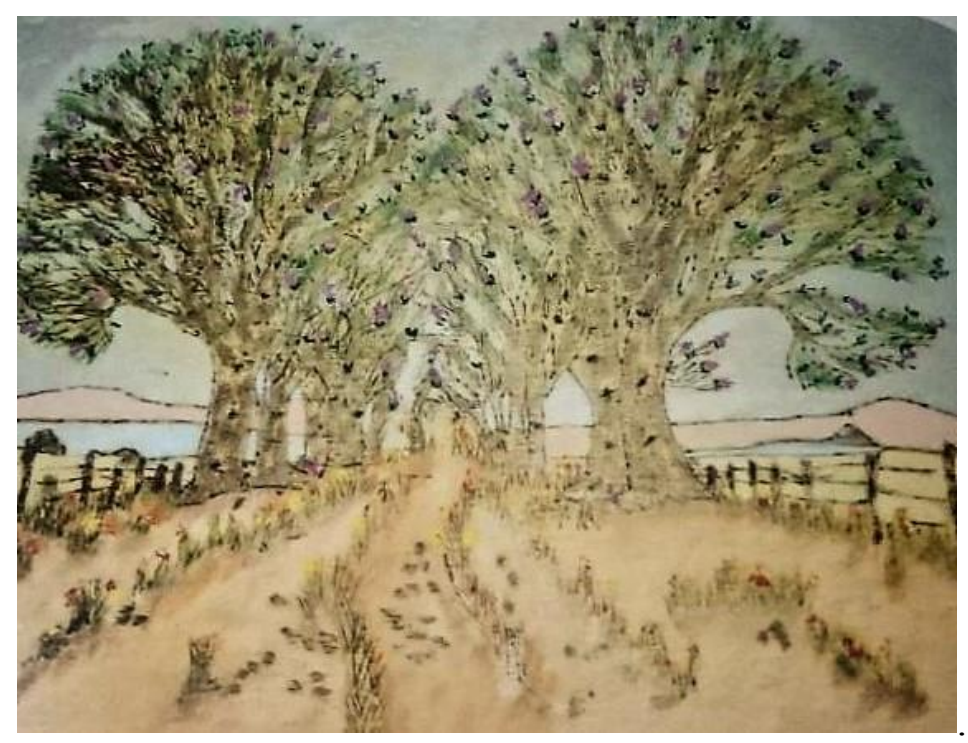

Ce tableau ${ }^{11}$ symbolise l'arbre de la vie, avec ses ramifications et sa riche floraison, nous pouvons le considérer comme un hymne à la fertilité et à la régénérescence de la nature.

\section{4- Rendre hommage au patrimoine immatériel marocain}

Ce n'est un secret pour personne, les femmes, de par le monde, sont gardiennes du patrimoine culturel et du savoir-faire. Un nombre important de tableaux de cet ouvrage représente des

\footnotetext{
${ }^{11}$ Ibid., p.46, de l'artiste Bahia Boukili
} 
aspects de ce patrimoine: les tenues traditionnelles (chez les femmes et les hommes), les bijoux, les décors, les danseurs et chanteurs d'Ahidous ${ }^{12}$ et la Tbourida ${ }^{13}$ ou fantasia. Comme la majorité de ces peintres est d'origine amazighe, le choix est porté souvent sur le patrimoine amazigh avec ses belles tenues, ses bijoux légendaires, son architecture, ses formes artistiques du spectacle $\ldots$

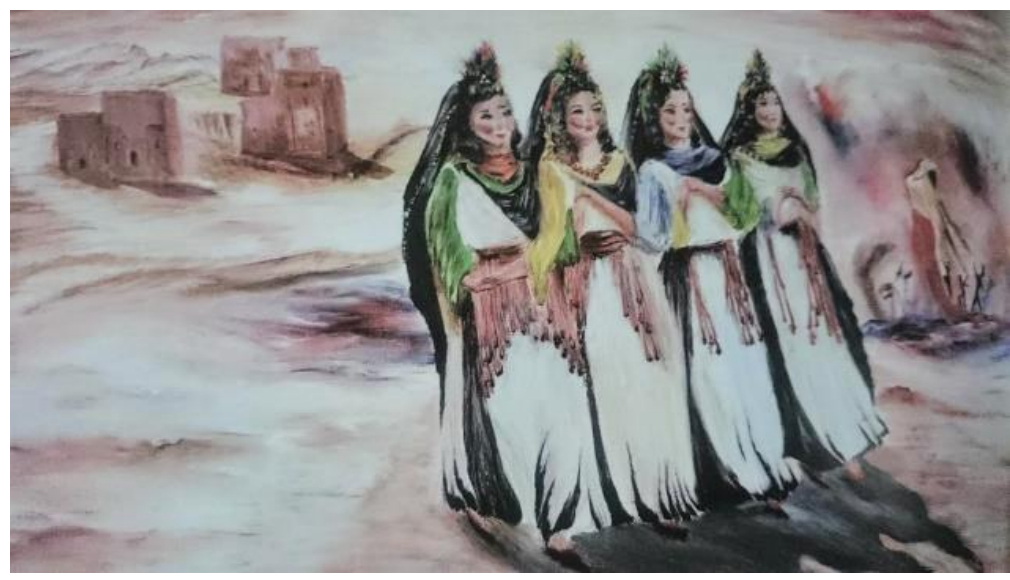

La jeune peintre Hasna Amghar, qui a exécuté ce tableau, est habitée par le désir de célébrer le patrimoine immatériel amazigh:

De tendance réaliste avec parfois une touche impressionniste, elles (ses toiles) sont le miroir de l'univers où Hasna a grandi. Par le choix des éléments qui peuplent l'espace pictural : architecture, oasis, palmiers, cérémonies de mariage, scènes de danse, de fantasia, des tenues traditionnelles, avec prédominance de la couleur ocre, ses tableaux ouvrent au regard une fenêtre sur le sud marocain avec ses dunes, ses vastes étendues et sa nudité. ${ }^{14}$

Le chant, la poésie, l'artisanat et le stylisme sont les autres formes artistiques qui s'inspirent et rendent hommage au patrimoine immatériel. Si nous commençons par considérer l'artisanat qui se sert d'éléments de la nature, la terre pour la poterie, la laine pour le tissage et la broderie, il est aisé de voir à quel point elles sont près de cette nature. Lors de la rencontre des

\footnotetext{
${ }^{12} L$ 'Ahidous est une forme d'art de spectacle du Maroc très répandu notamment au Maroc central et dans le sudest. L'Ahidous présente plusieurs variantes du Tafilalet (sud-est) jusqu'à Tifelt (nord-ouest). Il accompagne tous les événements festifs de ces régions qu'ils soient communautaires ou familiaux. Dans l'Ahidous, la femme occupe souvent un rôle central. Il est constitué de trois éléments essentiels : le chant lui-même dit izlan (pluriel de izli) se rapportant à la poésie locale ou d'une improvisation, le rythme musical principalement réalisé par des tambourins et les battements des mains, et les danse rassemblant hommes et femmes.

http://www.idpc.ma/view/pc immateriel/idpcm:A5DB3D
}

${ }^{13}$ La Tbourida, dérivée de Baroud qui signifie «poudre à canon », est un art équestre ancien, datant du 15 ème siècle. Elle est la reconstitution d'un assaut militaire de guerriers cavaliers arabes et berbères. De génération en génération, la Tbourida conserve une forte dimension spirituelle, notamment du fait qu'elle met le cheval, animal sacré de l'islam, au centre d'un spectacle haletant et impressionnant. La Tbourida éblouit le grand public marocain et international. Elle est associée aux festivités : moussems, fêtes agricoles et nombreuses fêtes nationales et familiales. Les internationaux la dénomment souvent «Fantasia », appellation d'origine latine, signifiant divertissement. https://www.sorec.ma/fr/la tbourida/

${ }^{14}$ Femmes artistes de Meknès-Tatilalet, op. cit., p.34. 
femmes artisanes qui vivent pour la plupart dans des villages isolés et en observant avec grand intérêt et admiration leurs productions artisanales,

Nous avons senti de la fierté chez toutes ces femmes modestes qui tissent, brodent et fabriquent non seulement pour vivre et parfois survivre mais aussi pour continuer à jouer leur rôle de dépositaires d'un patrimoine culturel de toute une région et de garantes de son identité. ${ }^{15}$

Elles reprennent les motifs déjà utilisés dans la peinture tels que les tenues et bijoux amazighs traditionnels :

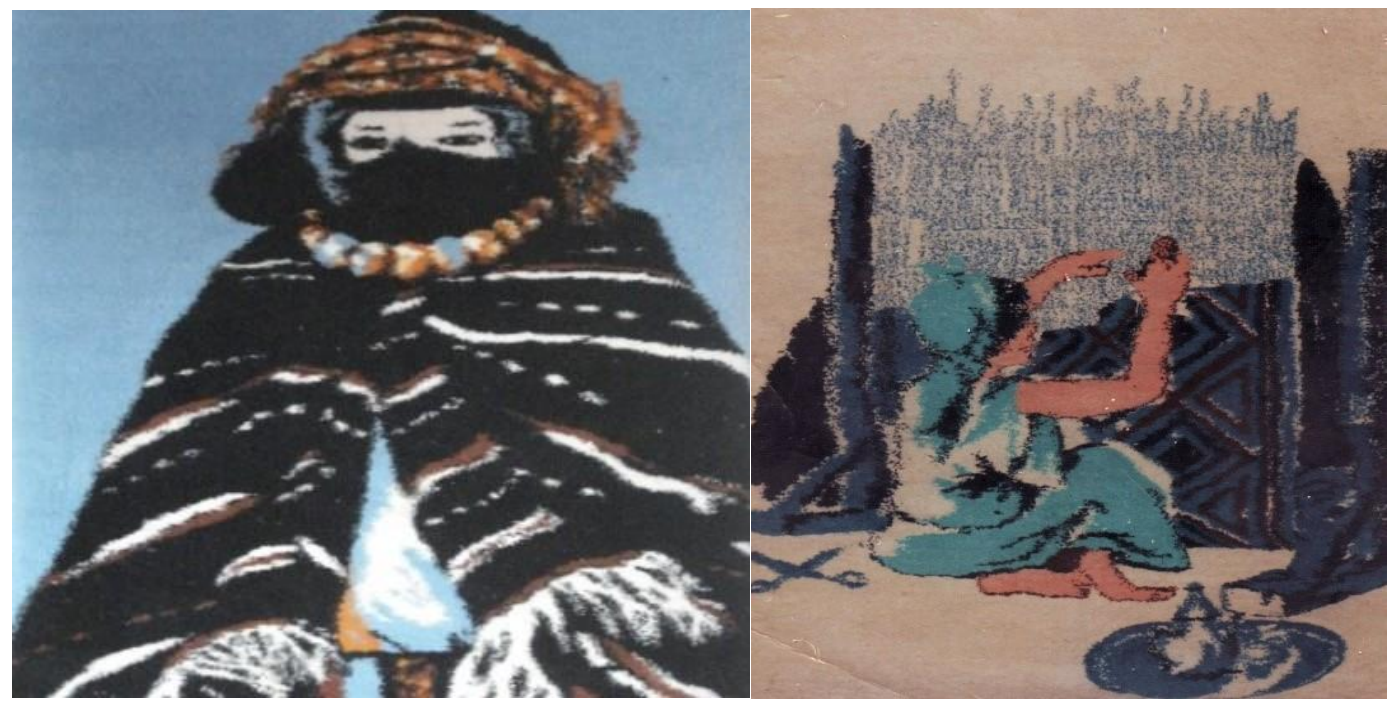

- $\quad$ Tapis tissés par des femmes du Moyen-Atlas

Le tapis ci-dessus où apparaît une tisseuse en train de tisser me fait penser à cette technique si répandue dans la littérature mais d'origine picturale (Les Ménines de Velasquez) appelée « la mise en abyme ». La tisseuse se tisse en train de tisser, ceci me fascine tout particulièrement venant d'une femme qui a su mettre au centre de sa création, de son tapis en l'occurrence, son métier de tissage. Innover est aussi leur désir de faire évoluer leur savoir-faire ancestral. Les plus jeunes n'hésitent pas à faire des recherches et des formations pour diversifier les thèmes et les matériaux, seule gageure pour perpétuer les traditions sans se départir de leur spécificité identitaire.

La poésie en amazigh est dite izlan, un genre oral par excellence qui participe à sauvegarder la mémoire sociale, celle de l'expérience humaine. «Les femmes la composent au retour des sources d'eau, lorsqu'elles collectent le bois, lorsqu'elles raillent les hommes, lorsqu'elles chantent l'amour, la vie, l'exil, la peur et les cris de la souffrance. $»^{16}$

\footnotetext{
${ }^{15}$ Femmes artistes de Meknès-Tafilalet, op.cit., , p.75.

16 lbid., 98.
} 
Nous avons eu la chance de rencontrer l'une des dernières poétesses amazighes maniant le verbe. Poétesse de l'oralité, Aïcha Othman Rakouchi, connue sous le nom de Taâtmant, a l'art de composer avec des mots hautement poétiques un hymne à la création, à la terre et à la tribu berbère. «Sa poésie est aussi une célébration des cycles de la vie, de la tolérance entre les hommes et un appel au respect du divin. Sa poésie rivalisait avec celle des hommes qu'elle réussissait à battre lors de joutes oratoires qu'elle garde précieusement dans sa mémoire et qui font sa fierté. $»^{17}$

Le chant amazigh à son tour exprime le rapport à la nature. L'artiste choisie pour représenter cette forme artistique est Chérifa Kerfit, la voix puissante du Moyen-Atlas. Elle est célèbre pour ses tamawayat, un genre de chant au rythme chevrotant et modulé issu, comme toute poésie amazighe, des forêts, des versants et des cimes des montagnes de son pays. Héritière d'une forme artistique distinguée du patrimoine ancestral, elle perpétue avec maestria la tradition de tamawayt aux côtés d'autres chanteuses célèbres telles que Tafrist, Oult Ouira et, la plus connue, Hadda Ouâkki.

Toutes ces femmes chanteuses ont dû briser les chaines et braver les tabous et les obstacles que la société dresse devant elles, les jugeant cruellement et les considérant comme des dépravées. Chanter devient dans ce cas un acte de bravoure et de courage les condamnant souvent à l'exclusion et à l'exil, non seulement loin de la famille mais aussi loin de la tribu. Une liberté chèrement payée.

\section{Conclusion}

Cette traversée du monde de l'art au féminin dans une région du Maroc n'a sans doute pas abordé tous les aspects du travail réalisé mais peut-être a-t-elle rapproché les lecteurs de la richesse de l'expérience artistique des femmes marocaines, des valeurs de leur culture et de leur patrimoine. Ces femmes sont en perpétuelle quête d'un centre, d'une identité et de nouvelles inspirations. Nous avons pu constater à quel point l'art est synonyme pour elles de liberté, d'expression de leur attachement à la vie, à la beauté, à la nature et aux valeurs humaines. L'art pour elles est également une prise de conscience de leur droit à la parole.

Cet ouvrage n'a d'autres objectifs que de rendre hommage à ces femmes artistes, humbles mais combien exceptionnelles, qui perpétuent et enrichissent le patrimoine immatériel, composante essentielle de l'identité culturelle de notre pays.

\footnotetext{
${ }^{17}$ Femmes artistes de Meknès-Tafilalet, op.cit., p.100
} 
Je conclus avec cette révélation de La Chana ${ }^{18}$, ex-reine de la danse flamenco, lors d'un entretien sur la chaîne de télévision Arte : «Quand je m'arrête de danser, c'est comme si on m'avait coupé les ailes. » Ceci résume parfaitement ce que l'art représente pour ces femmes, leurs ailes, leur force, leur univers.

\section{Annexes :}

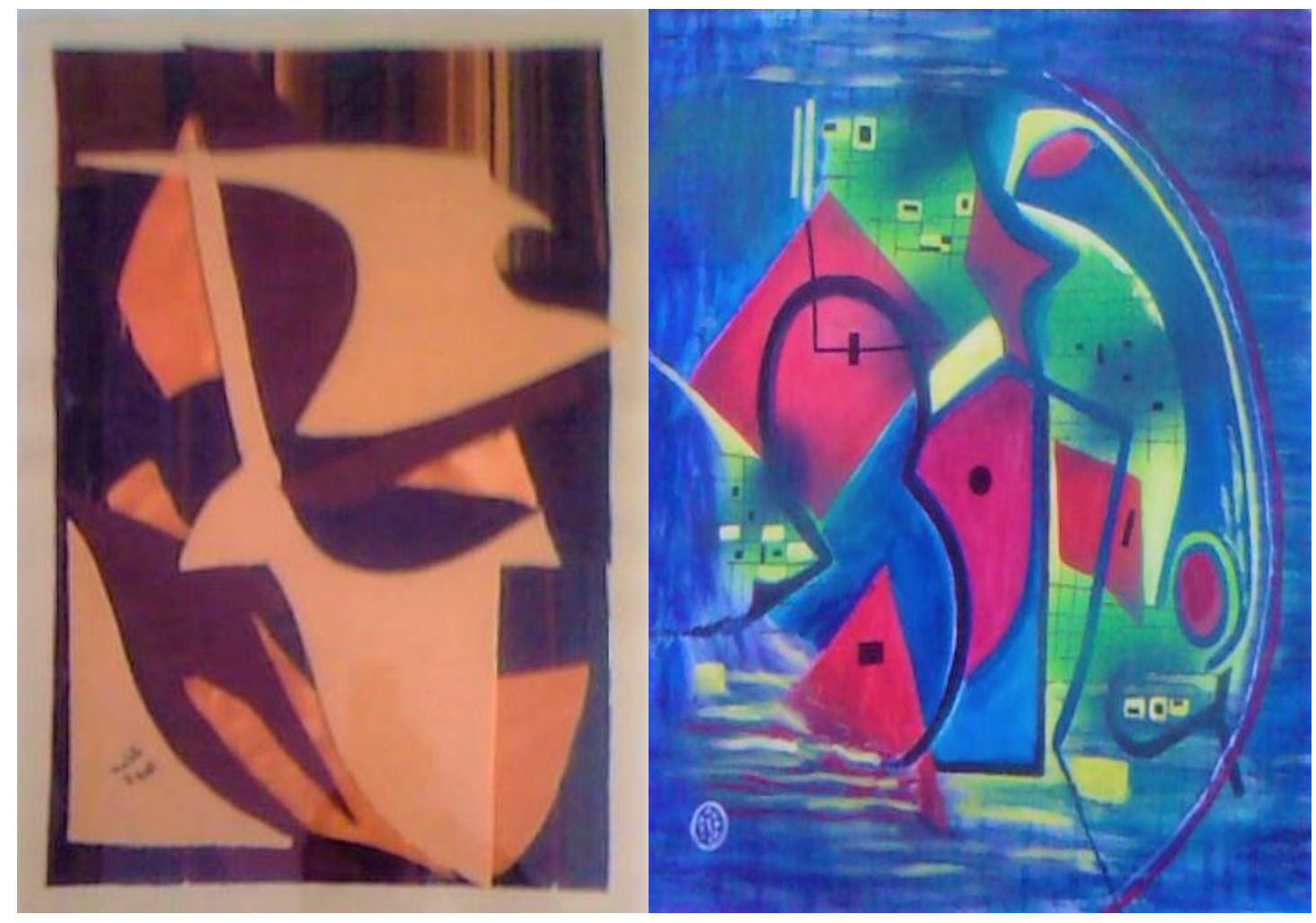

Tab. let 2 Chama Ami

\footnotetext{
${ }^{18}$ De son vrai nom Antonia Santiago Amador
} 


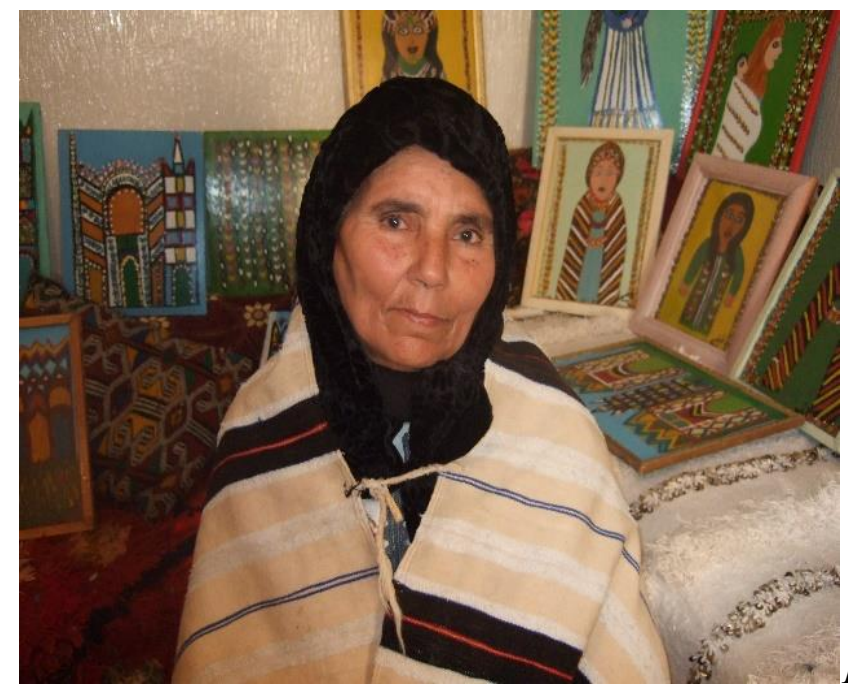

Fatima Benradouane dans son salon

transformé en salle d'exposition

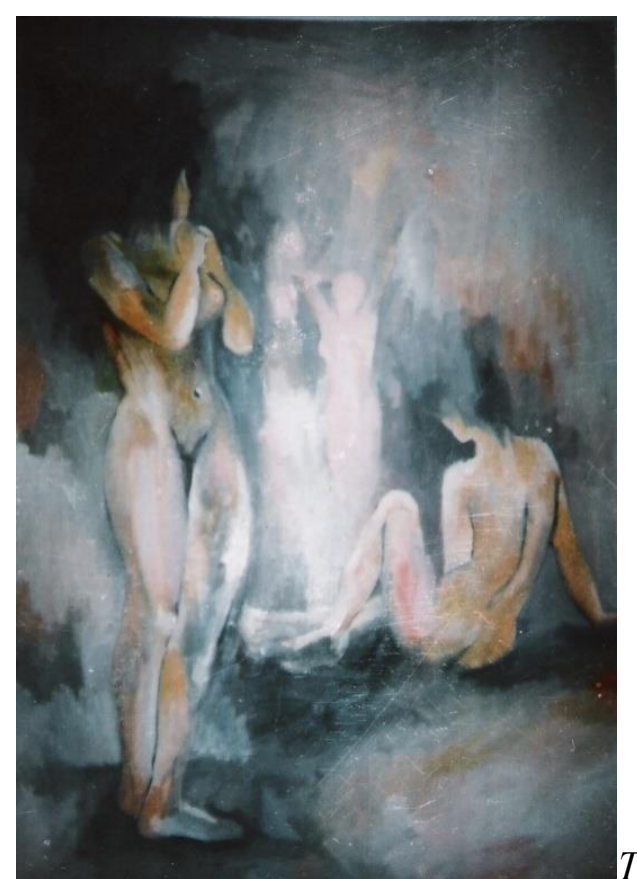

Tab.3. Zahra Awwaj, ce tableau n'a pas été publié dans le livre, la censure est passée par là, cependant, nous avons réussi à publier trois tableaux de cette artiste ainsi qu'un dessin. Voir ci-dessous. 


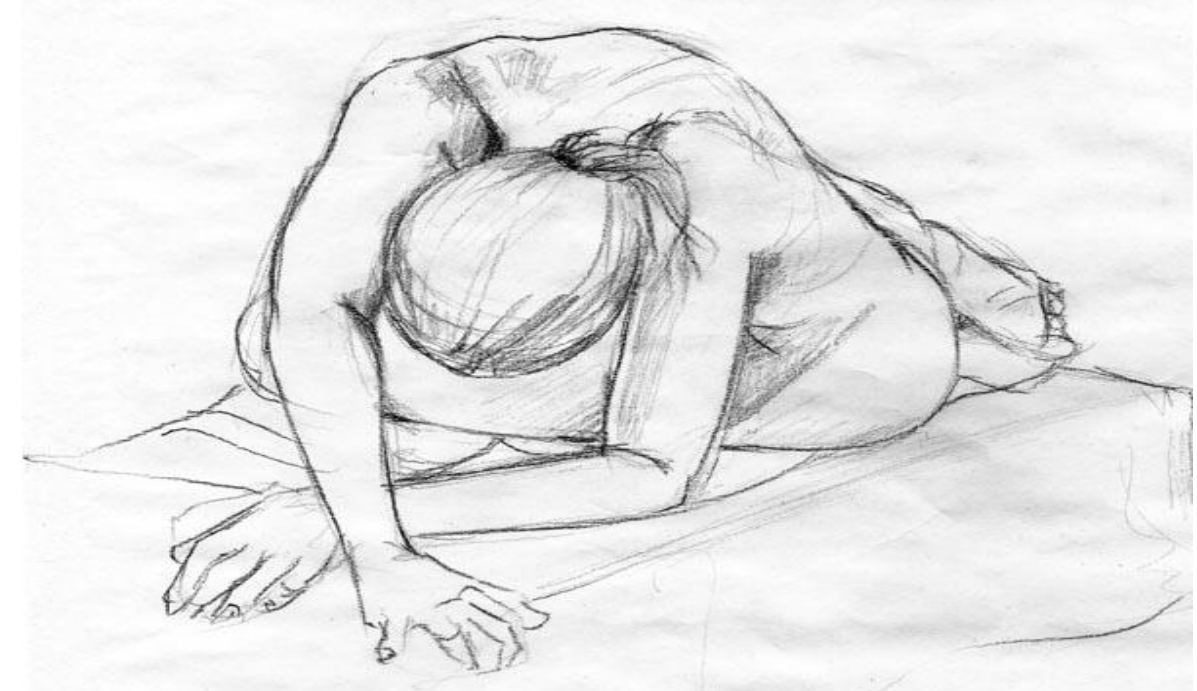

Tab. 4. Zahra Awwaj. Dessin

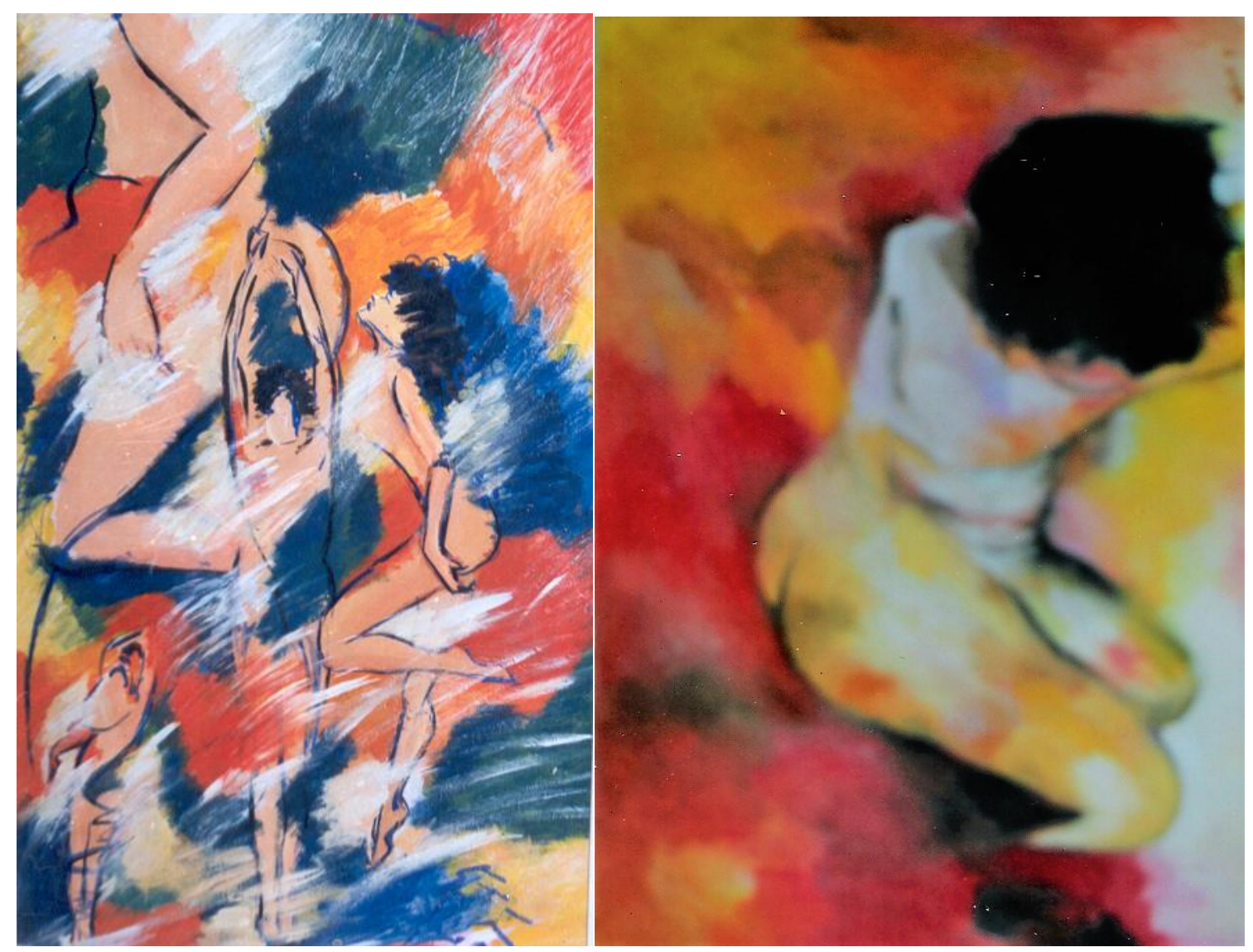

Tab.5 et 6. Zahra Awwaj. Nus 